\title{
Hinweise für Autoren
}

Bitte senden Sie Ihr Manuskript direkt an den Verlag S. Karger.

Es werden nur Originalarbeiten in deutscher Sprache veröffentlicht.

Das Manuskript soil in druckreifem Zustand in dreifacher Ausfertigung eingereicht werden. Die Entscheidung über die Aufnahme erfolgt aufgrund mehrerer Gutachten. Eine Kopie des Manuskripts soil grundsätzlich beim Autor verbleiben.

Die typographische Gestaltung und redaktionelle Bearbeitung ist dem

Verlag vorbehalten.

Autorkorrekturen über 10\% der Satzkosten hat der Autor zu tragen.

Dem Autor stehen 40 Gratissonderdrucke seiner Arbeit zu.

Verlagsrecht. Mit der Annahme des Manuskriptes gehen alle Rechte an Sonder- und

Nachdrucken, an Abdrucken in anderen Publikatio-nen sowie an Übersetzungen auf den Verlag über. Ebenso erhält der Verlag das Recht von Vervielfältigungen (auch von Abbildungen) jeder Art wie auch Vortrag, Funk, Fernsehen sowie Speicherung in Datenverarbeitungsanlagen - auch von Teilen eines Manuskripts.

Markennamen müssen, wenn sie geschützt sind, mit einem hochge-stellten ${ }^{\circledR}$ bezeichnet werden. Die Verantwortung für die korrekte Befolgung dieser Vorschrift liegt ausschließlich beim Autor. Abkürzungen sollen möglichst sparsam verwendet werden. Bei spe-ziellen Abkürzungen sollte der Autor jeweils beim ersten Auftreten das Wort ausschreiben und die später verwendete Abkürzung in Klammern folgen lassen: z.B. Adenosin-monophosphat (AMP).

Institutsangabe in der Sprache der Arbeit.

Zusammenfassungen sollen 20 Schreibmaschinenzeilen nicht über-schreiten. Eine Fassung in englischer Sprache und der englische Titel der Arbeit müssen mitgeliefert werden, auch wenn die Arbeit in deutscher Sprache abgefaßt ist. In die Zusammenfassung gehören keine Literaturzitate, Tabellen, Abbildungen und Abbildungshinweise sowie allgemein gehaltene Aussagen.

Schlüsselwörter sollen ebenfalls in deutscher und englischer Sprache abgefaßt werden. Zu einer Arbeit gehören drei bis fünf Key Words.

Tabellen, Abbildungen und Literaturzitate müssen im Text erwähnt werden.

Abbildungsvorlagen dürfen nicht aufgeklebt, gelocht oder mit Büro-klammern angeheftet eingereicht werden und sollen auf der Rückseite den Namen des Autors, den Titel des Beitrags sowie die Bildnummer tragen.

Adresse des Autors ist am Schluß der Arbeit vollständig anzugeben.

Literaturverzeichnis. Es soil nur die im Text zitierten Publikationen, diese dafür lückenlos, enthalten. Die Literatur ist entweder alphabe-tisch zu ordnen oder entsprechend der Reihenfolge im Text zu nume-rieren.

Kreiskrankenhaus Lemgo

- Schwerpunktkrankenhaus

mit 588 Betten -

Zum 1. Januar 1983 ist die Stelle eines Chefarztes für die Medizin. Abteilung II zu besetzen. 
Diese Abteilung wird zum 1. Januar 1983 neu mit 70-80 Betten eingerichtet. 15-20 Betten dieser Abteilung sind für onkologische Patienten vorgesehen.

Gesucht wird ein Internist. Vorausgesetzt wird eine langjährige und breite Erfahrung im Bereich der Diagnose, Therapie und

Nachsorge von Tumorerkrankungen mit hämatologischem/onkologischem Schwerpunkt.

Wünschenswert ist, daß die

klinischen Erfahrungen durch eine wissenschaftliche Qualifikation ergänzt sind und daß der Bewerber zu einer intensiven

interdisziplinären Zusammenarbeit auch mit den anderen Krankenhäusern des Kreises Lippe bereit ist.

Das Schwerpunktkrankenhaus Lemgo verfügt über hochqualifizierte diagnostische und therapeutische Einrichtungen und ist

zur Zeit in 10 hauptamtliche Fachabteilungen gegliedert:

Anästhesiologie, Chirurgie, Dermatologie, Gynäkologie und Geburtshilfe, Innere Medizin, Laboratoriumsmedizin,

Neurologie, Nuklearmedizin, Radiologie, Unfallchirurgie. Belegabteilungen: Augen und HalsNasen-Ohren.

Die Einstellung erfolgt auf der Basis des Nordrhein-Westfälischen Krankenhausgesetzes mit Privatdienstvertrag, Liquidationsrecht bei Anwendung eines Pool-Systems für die nachgeordneten Ärzte, beamtenrechtliche Versorgung oder als Zeitbeamter.

Lemgo selbst bietet einen hohen Wohnwert mit alien schulischen Möglichkeiten.

Ihre Bewerbung richten Sie bitte mit den üblichen Unterlagen einschließlich Ihrer wissenschaftlichen Veröffentlichungen und der Angabe von Referenzen bis drei Wochen nach Erscheinen dieser Anzeige an den

Oberkreisdirektor

- persönlich -

Postfach 89, 4930 Detmold 